\title{
Multiple power allocation for energy efficiency improvement in cognitive radio
}

\author{
Bing Ning, Shouyi Yang, Wanming Hao, Dawei Zang \\ School of Information Engineering \\ Zhengzhou University \\ Zhengzhou, China \\ ningbing0106@gmail.com
}

\begin{abstract}
This paper investigates the energy efficiency of sensing based spectrum sharing in cognitive radio network, where a secondary network can utilizes the primary network based the results of spectrum sensing. In the conventional sensing model, primary user is considered to remain active or inactive during the sensing slot. However, numerical results show that primary user could arrive at or depart from the band during the sensing slot of SU. A new three powers allocation strategy is proposed according to the effect of primary traffic. The resource allocation strategy is designed for the optimal energy-efficiency under the transmit power constraint and interference power constraint. Simulation results show that the energy efficiency of the proposed three power allocation is significantly improved compared with that of the conventional strategy.
\end{abstract}

Keywords-CR; energy efficiency; power allocation; sensing.

\section{INTRODUCTION}

This template, modified in MS Word 2007 and saved as a "Word 97-2003 Document" for the PC, provides authors with most of the formatting specifications needed for preparing electronic versions of their papers. Cognitive radio [1] is (CR) regarded as one promising technology to efficiently increase the utilization of the available radio spectrum. Recently, more and more research has focus on the energy-efficient wireless communication techniques [2] because energy efficiency is increasingly important for mobile devices.

In general, there are three types of operation for cognitive radio network: opportunistic spectrum access[3], spectrum sharing[4], and sensing based spectrum sharing[5][6]. Opportunistic spectrum access is a sensing based technology where secondary user (SU) should opportunistically access the licensed frequency band when primary user (PU) is detected to be inactive. Spectrum sharing allows SU coexist with PU when the interference to PU is tolerable. The sensing based spectrum sharing is a new model, where SU could adapt the transmit power to access the licensed band based spectrum sensing detection. Compared with the other types of operation, sensing based spectrum sharing is more flexible and dynamic to allocate system resource. In [5] and [6], the optimal resource allocation strategy is designed for the maximum throughput. During the sensing slot, PU is assumed to be either active or inactive. In reality, PU may depart from or arrive at the licensed band at any time, especially when the primary network has a high traffic rate or when a long

This work is supported by the National Nature Science Foundation of China (No.61271421). secondary frame interval is used. In [7], a more realistic CR network scenario is established that the primary user traffic during the sensing slot is considered. However, the corresponding power allocation is not studied for the optimal energy efficiency.

In this paper, we optimize the sensing time and power allocation strategy to obtain the optimal energy efficiency in the sensing based spectrum sharing cognitive radio network. The effect of primary user traffic during the sensing slot is considered. If PU is firstly active, then depart from the licensed band or firstly inactive, then arrive at the licensed band during the sensing slot, PU could be considered to remain inactive or active during the transmission slot, and SU could use the high power or low power; If PU remain active or inactive during the sensing slot, PU may depart from or arrive at the licensed band, then SU could use middle power to transmit during the transmit slot. Simulation results show that the new proposed power allocation strategy outperforms the conventional power allocation strategy in energy efficiency.

\section{SYSTEM MODEL}

A cognitive radio network (CRN) consists of a SU transmitter (SU-TX) and a SU receiver (SU-RX). The SU-TX accesses the licensed band based on the sensing results. The licensed band is occupied by one primary user(PU). The frame structure of SU consisting of a sensing slot and a transmission slot is shown in Fig.1. Let $g_{s s}, g_{s p}, g_{p s}$ denote the instantaneous channel gains of the secondary link, the link between SU transmitter and PU receiver, and the link between PU transmitter and SU receiver. At any time instant, the band is busy with probability $p_{0}=\alpha /(\alpha+\beta)$ and idle with probability $p_{1}=1-p_{0}$. The transition probability that the band is in state $\phi \in\{0,1\}$ given that seconds ago it was in state $\theta \in\{0,1\}$ is formulated as:

$$
\begin{aligned}
p_{\theta \phi}\left(T_{s}\right) & =\left(\begin{array}{ll}
p_{00}\left(T_{s}\right) & p_{01}\left(T_{s}\right) \\
p_{10}\left(T_{s}\right) & p_{11}\left(T_{s}\right)
\end{array}\right) \\
& =\frac{1}{\alpha+\beta}\left(\begin{array}{ll}
\alpha+\beta e^{-(\alpha+\beta) T_{s}} & \beta-\beta e^{-(\alpha+\beta) T_{s}} \\
\alpha-\alpha e^{-(\alpha+\beta) T_{s}} & \beta+\alpha e^{-(\alpha+\beta) T_{s}}
\end{array}\right)
\end{aligned}
$$




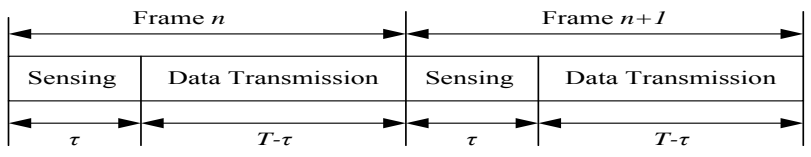

Fig. 1. Frame structure of the cognitive radio networks.

For simplify, we assume that the primary user state transition happens at most once within each frame or that the frame duration of PU is longer than the frame duration of SU. The PU could depart and arrive at the licensed band during the sensing slot of SU. There could be four cases according to the status of PU as follows:

$$
Y=\left\{\begin{array}{lr}
\sum_{i=1}^{I} n_{i}^{2} & , H_{0,0} \\
\sum_{i=1}^{d}\left(s_{i}+n_{i}\right)^{2}+\sum_{i=d+1}^{I} n_{i}^{2}, H_{0,1} \\
\sum_{i=1}^{I}\left(s_{i}+n_{i}\right)^{2} & , H_{1,0} \\
\sum_{i=1}^{a} n_{i}^{2}+\sum_{i=a+1}^{I}\left(s_{i}+n_{i}\right)^{2}, H_{1,1}
\end{array}\right.
$$

Where $H_{0,0}, H_{1,1}$ are the cases that PU is always absent and present during the sensing slot equivalent to the case in the conventional model [2]. $H_{0,1}$ is the case that PU is present for d samples and then departs from the licensed band during the sensing slot. $H_{1,0}$ is the case that PU is initially absent for $\mathrm{c}$ samples and then arrives at the licensed band during the sensing slot.

During the sensing slot, SU performs the detection on the licensed band occupied by PU. Accordingly the final detection on whether the band is busy or not will be dependent on the effect of PU's traffic in this model. Hence, the unconditional probabilities of false alarm and detection can be expressed as:

$$
\begin{aligned}
P_{f a N}(\eta, I) & =\frac{P\left(H_{0,1}, T_{s}\right) P_{f a H_{0,1}}(\eta, I)}{P\left(H_{0,1}, T_{s}\right)+P\left(H_{0,2}, T_{s}\right)} \\
& +\sum_{d=1}^{I}\left(\frac{p_{b} p_{11}^{d}\left(T_{s}\right) p_{10}\left(T_{s}\right) p_{00}^{J-d-1}\left(T_{S}\right)}{P\left(H_{0,1}, T_{s}\right)+P\left(H_{0,2}, T_{s}\right)} \cdot P_{f a H_{0,2}}(\eta, I, d)\right) \\
P_{d N}(\eta, I) & =\frac{P\left(H_{1,1}, T_{s}\right) P_{d H_{0,1}}(\eta, I)}{P\left(H_{1,1}, T_{s}\right)+P\left(H_{1,2}, T_{s}\right)} \\
& +\sum_{a=1}^{I}\left(\frac{p_{b} p_{11}^{a}\left(T_{s}\right) p_{01}\left(T_{S}\right) p_{11}^{J-a-1}\left(T_{S}\right)}{P\left(H_{1,1}, T_{s}\right)+P\left(H_{1,2}, T_{s}\right)} \cdot P_{d H_{1,2}}(\eta, I, a)\right)
\end{aligned}
$$

Where $P\left(H_{i, i}, T_{s}\right)$ denote the probability of each case occurring in the model, $P_{f a H_{i, j}}(\eta, I), P_{d H_{i, i}}(\eta, I)$ represent the condition probabilities of false alarm and detection, $i \in\{0,1\}$ (the expression of each probability can be found in [7]).

\section{THREE POWER ALLOCATIONS BASED SENSING RESULTS}

In the sensing based spectrum sharing model, SU detects the status of PU according to the effect of PU traffic,. If the case $H_{0,0}$ happens, PU may be always absent $h_{0}$ or arrive at the band $h_{1}$ during the transmission slot. In order to avoid the interference due to PU's arriving, SU can transmit with middle power $P_{s}^{m}$. If the cases $H_{0,1}$ and $H_{1,1}$ happen, PU could be considered to remain absent $h_{0}$ or present $h_{1}$ during the transmission slot. Then SU can transmit data with high power $P_{s}^{h}$ or low power $P_{s}^{l}$. If the case $H_{1,0}$ happens, $\mathrm{PU}$ may be always present $h_{1}$ or depart from the band $h_{0}$ during the

\begin{tabular}{|c|c|c|}
\hline Detection & $\begin{array}{l}\text { PU status } \\
\text { in }(T-\tau)\end{array}$ & SU Rate \\
\hline$H_{0,0}$ & $\begin{array}{c}h_{0} \\
h_{0} \rightarrow h_{1}\end{array}$ & $\begin{aligned} R_{0,0}\left(P_{s}^{m}\right) & =v_{0,0} r_{0,0}(J) \\
& +\sum_{a=I+1}^{J} \omega_{0,0} r_{0,0}(a)\end{aligned}$ \\
\hline$H_{0,1}$ & $h_{0}$ & $R_{0,1}\left(P_{s}^{h}\right)=P\left(H_{0,1}\right) r_{01}$ \\
\hline$H_{1,0}$ & $\begin{array}{c}h_{1} \\
h_{1} \rightarrow h_{0}\end{array}$ & $\begin{aligned} R_{1,0}\left(P_{s}^{m}\right) & =v_{1,0} r_{1,0}(J) \\
& +\sum_{d=I+1}^{J} \omega_{1,0} r_{1,0}(d)\end{aligned}$ \\
\hline$H_{1,1}$ & $h_{1}$ & $R_{1,1}\left(P_{s}^{l}\right)=P\left(H_{1,1}\right) r_{11}$ \\
\hline
\end{tabular}
transmission slot. In order to improve the effect of SU's throughput, SU can transmit with $P_{s}^{m},\left(P_{s}^{l}<P_{s}^{m}<P_{s}^{h}\right)$. The four possible scenarios are listed in Tab.1.

TABLE I THREE POWER ALLOCATION SCENARIOS FOR THE SBSS MODEL.

Where

$v_{0,0}=p_{e} p_{00}^{J}\left(T_{S}\right), v_{1,0}=p_{b} p_{11}^{J}\left(T_{s}\right)$

$\omega_{0,0}=p_{e} p_{00}^{a}\left(T_{s}\right) p_{01}\left(T_{s}\right) p_{11}^{J-1-a}\left(T_{s}\right)$

$\omega_{1,0}=\sum_{d=I+1}^{J-1} p_{b} p_{11}^{d}\left(T_{s}\right) p_{10}\left(T_{s}\right) P_{00}^{J-1-d}\left(T_{s}\right)$

$r_{01}=\log _{2}\left(1+\frac{g_{s s} P_{s}^{h}}{N_{0}}\right), r_{11}=\log _{2}\left(1+\frac{g_{s s} P_{s}^{l}}{g_{p s} P_{u}+N_{0}}\right)$

$r_{00}(a)=\log _{2}\left(1+\frac{g_{s s} P_{s}^{m}}{N_{0}+g_{p s} \frac{J-a}{J-I} P_{u}}\right), I+1 \leq a \leq J$

$r_{10}(d)=\log _{2}\left(1+\frac{g_{s s} P_{s}^{m}}{N_{0}+g_{p s} \frac{d-I}{J-I} P_{u}}\right), I+1 \leq d \leq J$

Accordingly, let $\alpha_{0}$ denote $1-P_{\text {faN }}(\eta, I)$ and $\beta_{0}$ denote $1-P_{d N}(\eta, I)$, the average achievable throughput for the CR network can be derived as: 


$$
\begin{aligned}
R\left(\tau, P_{s}^{l}, P_{s}^{m}, P_{s}^{h}\right) & =\frac{T-\tau}{T}\left[\alpha_{0}\left(R_{0,0}\left(P_{s}^{m}\right)+R_{0,1}\left(P_{s}^{h}\right)\right)\right. \\
& \left.+\beta_{0}\left(R_{1,0}\left(P_{s}^{m}\right)+R_{1,1}\left(P_{s}^{l}\right)\right)\right]
\end{aligned}
$$

In the transmission slot, the energy consumption for transmission at $\mathrm{SU}$ is :

$$
\begin{aligned}
\mathrm{E}_{c}\left(\tau, P_{s}^{l}, P_{s}^{m}, P_{s}^{h}\right) & =(T-\tau)\left\{\alpha_{0} P\left(H_{0,1}\right) P_{s}^{h}+\beta_{0} P\left(H_{1,1}\right) P_{s}^{l}\right. \\
& \left.+\left[\alpha_{0}\left(v_{0,0}+\omega_{0,0}\right)+\beta_{0}\left(v_{1,0}+\omega_{1,0}\right)\right] P_{s}^{m}\right\}
\end{aligned}
$$

Then the energy efficiency for the sensing based spectrum sharing CR system with the metric "Bit per Joule" is:

$$
U_{E E}\left(\tau, P_{s}^{l}, P_{s}^{m}, P_{s}^{h}\right)=\frac{R\left(\tau, P_{s}^{l}, P_{s}^{m}, P_{s}^{h}\right)}{\mathrm{E}_{c}\left(\tau, P_{s}^{l}, P_{s}^{m}, P_{s}^{h}\right)+\mathrm{E}_{s}}
$$

Where $\mathrm{E}_{s}$ is the circuit power consumption derived from signal processing, battery backup, and others.

$P_{a v}$ denotes the maximum average transmission power of SU. The transmission power constraint can be expressed as:

$$
\begin{aligned}
& \frac{T-\tau}{T} E\left\{\alpha_{0} P\left(H_{0,1}\right) P_{s}^{h}+\beta_{0} P\left(H_{1,1}\right) P_{s}^{l}\right. \\
& \left.+\left[\alpha_{0}\left(v_{0,0}+\omega_{0,0}\right)+\beta_{0}\left(v_{1,0}+\omega_{1,0}\right)\right] P_{s}^{m}\right\} \leq P_{a v}
\end{aligned}
$$

When SU is active, the primary link will suffer from the potential inter-interference. As a result, As a result, the interference power constraint can be defined as:

$$
\begin{aligned}
\frac{T-\tau}{T} E\left\{\left[\alpha_{0}\left(v_{0,0}+\omega_{0,0}\right)\right.\right. & \left.+\beta_{0}\left(v_{1,0}+\omega_{1,0}\right)\right] g_{s p} P_{s}^{m} \\
& \left.+\beta_{0} P\left(H_{1,1}\right) g_{s p} P_{s}^{l}\right\} \leq \Gamma
\end{aligned}
$$

where $\Gamma$ is the maximum tolerable interference power at PU.

Accordingly, the energy-efficiency power allocation problem of the sensing-based spectrum sharing CR system can be written as:

$$
\max _{\tau, P_{s}^{l}, P_{s}^{m}, P_{s}^{h}} U_{E E}\left(\tau, P_{s}^{l}, P_{s}^{m}, P_{s}^{h}\right)
$$

Subject to (6),(7), $0 \leq \tau \leq \mathrm{T}, P_{s}^{h} \geq 0, P_{s}^{m} \geq 0, P_{s}^{l} \geq 0$

The objective function is not convex with respect to the sensing time. Since $0 \leq \tau \leq \mathrm{T}$, the optimal sensing time can be obtained through one-dimensional exhaustive search:

$$
\tau_{\text {opt }}=\operatorname{argmax}_{\tau} U_{E E}\left(\tau, P_{s}^{l}, P_{s}^{m}, P_{s}^{h}\right)
$$

The optimal problem can be associated with the following function problem $\mathrm{F}(q)$ for fixed $\hat{\tau}$ :

$$
\max _{\hat{\tau}, P_{s}^{l}, P_{s}^{m}, P_{s}^{h}} R\left(\hat{\tau}^{\wedge} P_{s}^{l}, P_{s}^{m}, P_{s}^{h}\right)-q\left(\mathrm{E}_{c}\left(\tau, P_{s}^{l}, P_{s}^{m}, P_{s}^{h}\right)+\mathrm{E}_{s}\right)
$$

where $q \in R$ is a parameter.
Theorem 1. $\left(P_{s}^{l, *}, P_{s}^{m, *}, P_{s}^{h, *}\right)$ is the optimal solution of the objective problem associated with the maximum value $q^{*}$ if and only if $\mathrm{F}\left(q^{*}\right)=\mathrm{F}\left(q^{*}, P_{s}^{l, *}, P_{s}^{m,{ }^{*}}, P_{s}^{h, *}\right)=0$.

Prove: If $\left(P_{s}^{l, *{ }^{*}}, P_{s}^{m, *}, P_{s}^{h, *}\right)$ is the optimal solution of the problem associated with the maximum value $q^{*}$, then:

$$
q^{*}=\frac{R\left(\hat{\tau}, P_{s}^{l, *}, P_{s}^{m, *^{*}}, P_{s}^{h, *}\right)}{\mathrm{E}_{c}\left(\hat{\tau}, P_{s}^{l, *}, P_{s}^{m, *}, P_{s}^{h, *}\right)+\mathrm{E}_{s}}
$$

This equation implies that:

$R\left(P_{s}^{l, *}, P_{s}^{m,{ }^{*}}, P_{s}^{h, *}\right)-q^{*}\left(\mathrm{E}_{c}\left(P_{s}^{l, *}, P_{s}^{m, *}, P_{s}^{h, *}\right)+\mathrm{E}_{c}\right)=0$

$R\left(P_{s}^{l}, P_{s}^{m}, P_{s}^{h}\right)-q^{*}\left(\mathrm{E}_{c}\left(P_{s}^{l}, P_{s}^{m}, P_{s}^{h}\right)+\mathrm{E}_{c}\right) \leq 0$

Then conclude $\mathrm{F}\left(q^{*}\right)=\mathrm{F}\left(q^{*}, P_{s}^{l, *}, P_{s}^{m, *}, P_{s}^{h, *}\right)=0$.

$\operatorname{IfF}\left(q^{*}\right)=\mathrm{F}\left(q^{*}, P_{s}^{l, *}, P_{s}^{m, *}, P_{s}^{h, *}\right)=0$, then :

$\frac{R\left(\hat{\tau}, P_{s}^{l, *}, P_{s}^{m, *}, P_{s}^{h, *}\right)}{\mathrm{E}_{c}\left(\hat{\tau}^{\wedge} P_{s}^{l, *}, P_{s}^{m, *}, P_{s}^{h, *}\right)+\mathrm{E}_{c}}=q^{*} ; \frac{R\left(\tau, P_{s}^{l}, P_{s}^{m}, P_{s}^{h}\right)}{\mathrm{E}_{c}\left(\tau, P_{s}^{l}, P_{s}^{m}, P_{s}^{h}\right)+\mathrm{E}_{c}} \leq q^{*}$

Thus $\left(P_{s}^{l, *}, P_{s}^{m, *}, P_{s}^{h, *^{*}}\right)$ is the optimal solution of the problem with the maximum value $q^{*}$.

The optimization problem is convex with respect to the power $\left(P_{s}^{l}, P_{s}^{m}, P_{s}^{h}\right)$. By employing the Lagrange dual decomposition method, the joint optimization problem could be decomposed into three sub-optimal problems:

$$
\begin{aligned}
S P 1: & \frac{T-\tau}{T} \alpha_{0} P\left(H_{0,1}\right)\left\{r_{01}-(q T+\lambda) P_{s}^{h}\right\} \\
S P 2: & \frac{T-\tau}{T}\left\{\alpha_{0}\left(v_{0,0} r_{0,0}(J)+\sum_{a=I+1}^{J} \omega_{0,0} r_{0,0}(a)\right)\right. \\
& +\beta_{0}\left(v_{1,0} r_{1,0}(J)+\sum_{d=I+1}^{J} \omega_{1,0} r_{1,0}(d)\right) \\
& \left.-\left(q T+\lambda+\mu g_{s p}\right)\left(\alpha_{0}\left(v_{0,0}+\omega_{0,0}\right)+\beta_{0}\left(v_{1,0}+\omega_{1,0}\right)\right) P_{s}^{m}\right\}
\end{aligned}
$$

SP3: $\frac{T-\tau}{T} \beta_{0} P\left(H_{1,1}\right)\left\{r_{11}-\left(q T+\lambda+\mu g_{s p}\right) P_{s}^{l}\right\}$

Where $\lambda$ is the dual variable associated with the transmit power constraint, and $\mu$ is the variable associated with the interference power constraint.

After applying the KKT condition, the optimal power allocation $\left(P_{s}^{l}, P_{s}^{m}, P_{s}^{h}\right)$ can be obtained as follows:

$$
\begin{aligned}
& P_{s}^{l}=\frac{1}{q T+\lambda+\mu g_{s p}}-\frac{g_{p s} P_{u}+N_{0}}{g_{s s}} \\
& P_{s}^{m}=\left(\frac{\alpha_{0}\left(v_{0,0}+(J-I-1) \omega_{0,0}\right)+\beta_{0}\left(v_{1,0}+(J-I-1) \omega_{1,0}\right)}{\left(q T+\lambda+\mu g_{s p}\right)\left(\alpha_{0}\left(v_{0,0}+\omega_{0,0}\right)+\beta_{0}\left(v_{1,0}+\omega_{1,0}\right)\right)}\right)^{\frac{1}{2}}
\end{aligned}
$$


$P_{s}^{h}=\frac{1}{q T+\lambda}-\frac{N_{0}}{g_{s s}}$

According to Theorem 1, obtaining the optimal power allocation is equivalent to finding the root for the equation, $\mathrm{F}\left(q^{*}\right)=\mathrm{F}\left(q^{*}, P_{s}^{l, *}, P_{s}^{m, *}, P_{s}^{h, *}\right)=0$. For fixed $\lambda$ and $\mu$, the bisection search method can be used to find the optimal $q^{*}$ and solve the optimization problem.

\section{Simulation RESUlts}

In this section, we numerically evaluate the optimal system performance of sensing based spectrum sharing cognitive radio networks. The frame duration of secondary systems is set as $\mathrm{T}=100 \mathrm{~ms}$, the target detection probability is chosen as $P_{d N}(\eta, I)=0.9$, and the noise variance is set to be 1 . The circuit power is set to be $0.4 \mathrm{w}$.

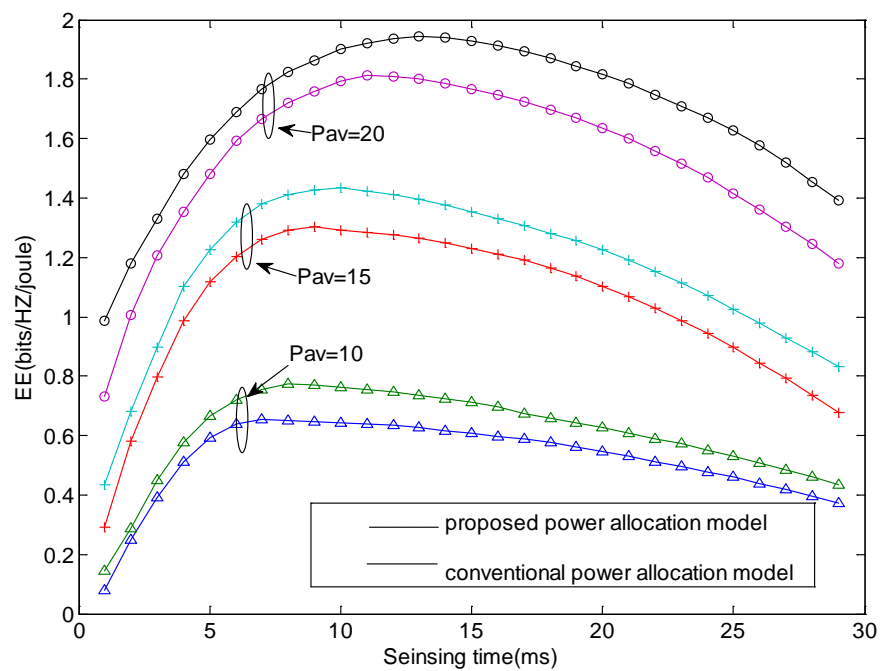

Fig. 2. Energy efficiency versus sensing time.

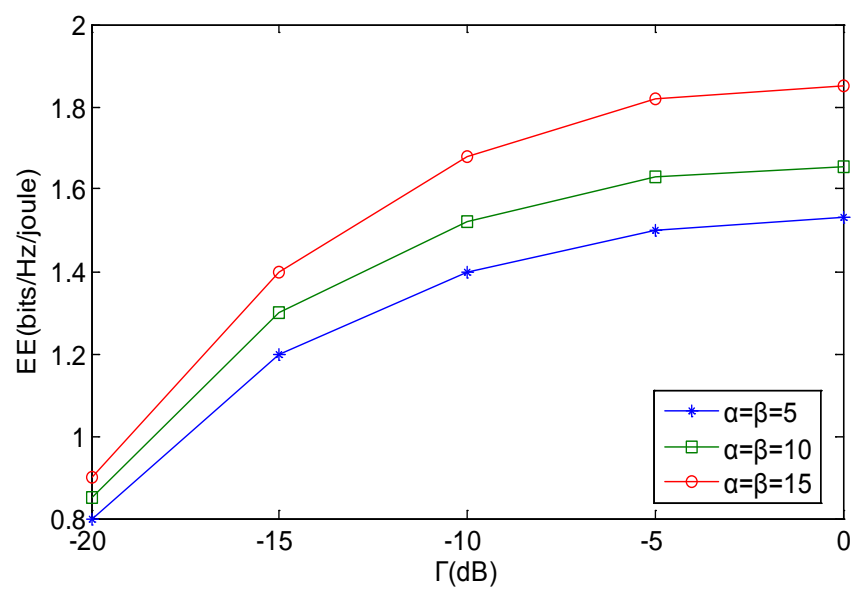

Fig. 3. Energy efficiency versus the interference power constraint
In Fig. 2, the energy efficiency of secondary user versus sensing time is presented for several values of the maximum average transmission power of SU. The maximum interference power is assumed as $\Gamma=-10 d B$. Fig. 2 shows the energy efficiency is a convex function of the sensing time. The maximum energy efficiency is obtained at the optimal sensing time. The energy efficiency in the proposed power allocation model is higher than that in the conventional model and increases with $P_{a v}$ increasing, which is due to the fact that the multiple power allocation allows more accurate strategy of data transmission in the new model.

In Fig. 3, the energy efficiency of the secondary base station versus the average interference power constraint $\Gamma$ is presented for different values $\alpha, \beta$. The sensing time is assumed to be the optimal sensing time, which is $10 \mathrm{~ms}$. Energy efficiency increases slowly when the interference power constraint becomes higher in value. This condition is reasonable because the interference power constraint is not the main limit for the maximum energy efficiency when the interference power receives higher values.

\section{CONCLUSION}

In this paper, we investigate the optimization problem of sensing time and multiple power allocation for the optimal energy efficiency in sensing based spectrum sharing CR networks. In the sensing slot, the random arrival and departure primary user traffic is considered. The detection results is decided based on the effect of primary user traffic. In the transmission slot, the multiple power allocation strategy is designed according to the status of PU during the sensing slot. Simulation results show that the multiple power allocation strategy outperforms that in the conventional model, where the effect of primary user traffic isn't considered.

\section{Reference}

[1] J.M.III and J.G.Q.Maguire, "Cognitive radios: making software radio more personal," IEEE Personal Commun, vol.6,no.4, pp,13-18, August1999.

[2] Guowang Miao, "Energy-Efficient uplink multi-user MIMO." IEEE Transactions on Wireless Communications, Vol.12, No.5, May 2013.

[3] Q.chen, Y.C.Liang, M.Motani, and W-C.Wong, "A two-level mac protocol strategy for opportunistic spectrum access in cognitive radio networks." IEEE Transactions on Vehicular Technology, vol.60, no.5, pp.2164-2180, June 2011.

[4] X.Kang, Y.C.Liang, A.Nallanathan, H.K.Garg and R.Zhang, "Optimal power allocation for OFDM-based cognitive radio with new primary transmission protect criteria." IEEE Transactions on Wireless Communications, vol.9, no.6, February 2010.

[5] S.Stotas and A.Nallanathan, "Optimal sensing time and power allocation in multiband cognitive radio networks." IEEE Transactions on Communications, vol.59, no.1, pp.226-235, January 2011.

[6] B.Ning, S. Yang, L.Liu, and Y.Lu, "Resource allocation for OFDM cognitive radio within enhanced primary transmission protection." IEEE Communications Letters, Vol.18, No.11, pp. 2027-2030, November 2014.

[7] Tang .L, Chen .Y.F and Alouini M.S. Effect of Primary User Traffic on Sensing-Throughput Tradeoff for Cognitive Radios[J].IEEE Trans.on Wireless Commun.,Apr. 2011,vol. 10, no. 4, pp. 1063-1068 